\title{
Three-Dimensional MIMO Channel Model with High-Mobility Wireless Communication Systems Using Leaky Coaxial Cable in Rectangular Tunnel
}

\author{
Kai Zhang $\left(\mathbb{D},{ }^{1}\right.$ Fangqi Zhang, ${ }^{2,3,4}$ Guoxin Zheng $\mathbb{D}^{1},{ }^{1}$ and Lei Cang ${ }^{1}$ \\ ${ }^{1}$ Key Laboratory of Speciality Fiber Optics and Optical Access Networks, Joint International Research Laboratory \\ of Speciality Fiber Optics and Advanced Communication, Shanghai University, Shanghai 200444, China \\ ${ }^{2}$ Key Laboratory of Road and Traffic Engineering, Tongji University, Ministry of Education, Shanghai 201804, China \\ ${ }^{3}$ Tongji University Maglev Transportation Engineering R\&D Center, Tongji University, Shanghai 201804, China \\ ${ }^{4}$ The Cooperative Center for Maglev and Transit Operation Control System, Tongji University, Shanghai 201804, China \\ Correspondence should be addressed to Guoxin Zheng; gxzheng@staff.shu.edu.cn
}

Received 19 November 2020; Revised 31 January 2021; Accepted 4 February 2021; Published 23 February 2021

Academic Editor: Jose-Maria Molina-Garcia-Pardo

Copyright (c) 2021 Kai Zhang et al. This is an open access article distributed under the Creative Commons Attribution License, which permits unrestricted use, distribution, and reproduction in any medium, provided the original work is properly cited.

\begin{abstract}
With the rapid development of high-mobility wireless communication systems, e.g., high-speed train (HST) and metro wireless communication systems, more and more attention has been paid to the wireless communication technology in tunnel-like scenarios. In this paper, we propose a three-dimensional (3D) nonstationary multiple-input multiple-output (MIMO) channel model with high-mobility wireless communication systems using leaky coaxial cable (LCX) inside a rectangular tunnel over the $1.8 \mathrm{GHz}$ band. Taking into account single-bounce scattering under line-of-sight (LoS) and non-line-of-sight (NLoS) propagations condition, the analytical expressions of the channel impulse response (CIR) and temporal correlation function (T-CF) are derived. In the proposed channel model, it is assumed that a large number of scatterers are randomly distributed on the sidewall of the tunnel and the roof of the tunnel. We analyze the impact of various model parameters, including LCX spacing, time separation, movement velocity of Rx, and K-factor, on the T-CF of the MIMO channel model. For HST, the results of some further studies on the maximum speed of $360 \mathrm{~km} / \mathrm{h}$ are given. By comparing the T-CF between the dipole MIMO system and the LCX-MIMO system, we can see that the performance of the LCX-MIMO system is better than that of the dipole MIMO system.
\end{abstract}

\section{Introduction}

Recently, high-mobility wireless communication systems have developed rapidly, for example, high-speed train (HST) and metro wireless communication systems. The speed of HST is getting faster and faster; for example, the trail maximum speed of HST reaches nearly $575 \mathrm{~km} / \mathrm{h}$ by the French National Rail Corporation, the maximum speed of Japan's seven-car "magnetic levitation train" reaches $603 \mathrm{~km} / \mathrm{h}$, China's CIT500 HST hits a train wheel of $605 \mathrm{~km} /$ $\mathrm{h}$, and the current operating speed of China's HST is $350 \mathrm{~km} /$ h. Leaky coaxial cable (LCX) is widely used in urban subway tunnel environments. LCX has many advantages; for example, its radiation characteristics provide uniform coverage, longitudinal attenuation along the axis of LCX is very small, and its installation is very simple [1-12]. Therefore, LCX is very suitable for the wireless communication system in a tunnel scenario. In addition, the multiple-input multiple-output (MIMO) technology is widely used in improving the spectrum efficiency and the propagation characteristics of wireless communication systems. In order to improve the stability and reliability of wireless communication systems in the tunnel environment, the MIMO using LCX technology has become an inevitable trend.

The previous research on LCX mainly includes the following: the characteristics analysis of LCX, for example, radiation characteristic [3]; the performance analysis of $2 \times 2$ MIMO system using single LCX in free space, e.g., channel capacity and correlation [1]; the study of MIMO system performance using LCX in tunnel scenario [2]; the 
performance of LCX-MIMO in an indoor office environment, e.g., channel capacity and channel power spread [4]; the statistics characterization of $2 \times 2$ MIMO system using single LCX in linear-cell environment, including capacity using different power allocation (i.e., equal power allocation and water-filling power allocation) over the $2.4 \mathrm{GHz}$ band and the $5 \mathrm{GHz}$ band [5], condition number $(\mathrm{CN})$ with different polarization types (i.e., vertical polarization and horizontal polarization) over wireless local area network (WLAN) frequency band (i.e., $2.4 \mathrm{GHz}$ ) [6,7]; and the performance of $4 \times 4$ MIMO system using two LCX in linear-cell environment, including capacity using different power allocation (i.e., equal power allocation and water-filling power allocation) with various LCX spacing over the $2.4 \mathrm{GHz}$ band and the $5 \mathrm{GHz}$ band [8], condition number $(\mathrm{CN})$ with different LCX spacing over the $5 \mathrm{GHz}$ band [9], and the $2.4 \mathrm{GHz}$ band [10]. In [11], the author proposed a LCX-MIMO channel model based on electric field distribution of LCX in tunnel, and capacity, $\mathrm{CN}$, and correlation are analyzed by simulation and measurement. In [12], the author proposed a nonstationary LCX-MIMO channel model based on electric field distribution of LCX and geometry-based stochastic model (GBSM) in the tunnel. The space-time correlation is analyzed by simulation. The velocities of movement are $90 \mathrm{~km} / \mathrm{h}, 180 \mathrm{~km} / \mathrm{h}$, and $360 \mathrm{~km} / \mathrm{h}$. According to the theory of electromagnetic field, with the increase of electromagnetic wave propagation distance, the electric field strength decreases gradually. The channel model in [12] is mainly based on the electric field distribution around the LCX, and only considering the change of the amplitude of the electromagnetic wave signal with the increase of the propagation path distance, but not considering that when the electromagnetic wave signal is scattered by the scatterer, it will not only introduce the phase shift by the scatterer but also attenuate the amplitude of the signal by the scatterer.

With the rapid development of wireless communication, more and more attention has been paid to the nonstationary MIMO fading channel in various scenarios. According to various scenarios, there are different channel models, including spatially and temporally correlated channel model for MIMO system in mobile fading channels [13-15] and mobile-to-mobile channels [16], 3D nonstationary MIMO channel model with three-dimensional (3D) antennas array and 3D arbitrary trajectories with mobile station [17], 3D nonstationary GBSM unmanned aerial vehicle (UAV) MIMO channel model for air-to-ground (A2G) [18] and wideband [19] wireless communication system, 3D nonstationary geometry-based MIMO channel model for vehicle-to-vehicle $(\mathrm{V} 2 \mathrm{~V})$ communication in the tunnel (i.e., semicircular tunnel and rectangular tunnel) environment [20-22], and geometry-based single-bounce (GBSB) channel model for MIMO system in tunnel [23]. In these channel models, the influence of different channel parameters, including the transmitter and receiver antennas spacing, the number of transmitter and receiver antennas, angle of arrival (AOA) and angle of departure (AOD), movement time of transmitter/receiver antennas, movement velocity of transmitter/receiver antennas, and time separation, on channel capacity and correlation is analyzed.
In summary, so much literature has analyzed the performance of the LCX-MIMO system. At the same time, much literature has proposed a large number of nonstationary channel models for various scenarios. However, there is a few of high-mobility channel model for LCX-MIMO wireless communication system in a tunnel environment.

In order to further study the performance of a highmobility wireless communication system using LCX-MIMO in a tunnel-like scenario, this paper proposes a $3 \mathrm{D}$ nonstationary LCX-MIMO channel model with a high-mobility wireless communication system in a rectangular tunnel based on GBSM, and we are considering that when the electromagnetic wave signal is scattered by the scatterer, it will not only introduce the phase shift by the scatterer but also attenuate the amplitude of the signal by the scatterer. The analytical expressions of the channel impulse response (CIR) and the temporal correlation function (T-CF) are derived. The utility and reasonability of the proposed channel model are verified by experiment and simulation. Meanwhile, we analyze the influence of various model parameters, including LCX spacing, time separation, movement velocity of $\mathrm{Rx}$ and $K$-factor, on the T-CF of the MIMO channel model by simulation. For HST, the results of some further studies on the maximum speed of $360 \mathrm{~km} / \mathrm{h}$ are given.

The remainder of this paper is organized as follows: In Section 2, the LCX-MIMO channel model is derived. In Section 3, the channel T-CF is derived theoretically, and the formula of capacity and $K$-factor are described. Section 4 is experiment results and analysis. Section 5 is simulation results and validation. Section 6 describes the conclusion of this paper.

\section{LCX-MIMO Channel Model}

In this paper, for the rectangular tunnel scenario, the transmitters (Tx) and receivers (Rx) of the LCX-MIMO communication system are consisting of two LCXs and two dipole antennas, respectively. In Figure 1, o represents the origin of the coordinate. The parallel direction to the axis of the tunnel is considered as the $z$-axis of the coordinate system. The cross section of the tunnel is in the xoy plane, the vertical direction of the plane is along the $y$-axis of the coordinate system, and, finally, the horizontal direction of the plane is along the $x$-axis of the coordinate system; the S1 and S2 are input signals of LCXs. Figure 1(a) describes the structure of LCX; Figure 1(b) describes the propagation scenario of MIMO using two LCXs in the tunnel; Figure 1(c) describes the nonstationary 3D LCXMIMO channel model in the tunnel, (-•-) denotes singlebounce non-line-of-sight $(\mathrm{NLoS})$ path, $(-\bullet-)$ denotes line-ofsight (LoS) paths; and Figure 1(d) describes the randomly distributed scatters in the tunnel.

Here, the slot period of LCX is set as $P, L$ is the length of LCX, and the number of slots is $N=$ floor $(L / P)-1$. According to the properties of LCX, the longitudinal attenuation factor of the $i$-th $(i=1, \ldots, N)$ slot of LCX is $a_{i}=\alpha_{i} e^{-j \beta_{i}}$, where the longitudinal amplitude attenuation of $i$-th slot is $\alpha_{i}=\alpha_{0}^{i}, \alpha_{0}$ is the longitudinal amplitude attenuation per unit period of slots, and $\beta_{i}$ is the phase variation of $i$-th slot. The LCX used in this paper refers to the LCX with a working frequency band of $1.8 \mathrm{GHz}$ provided by the 


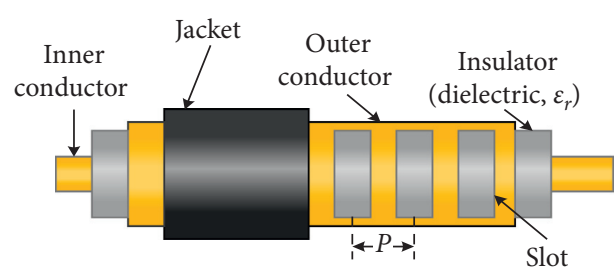

(a)

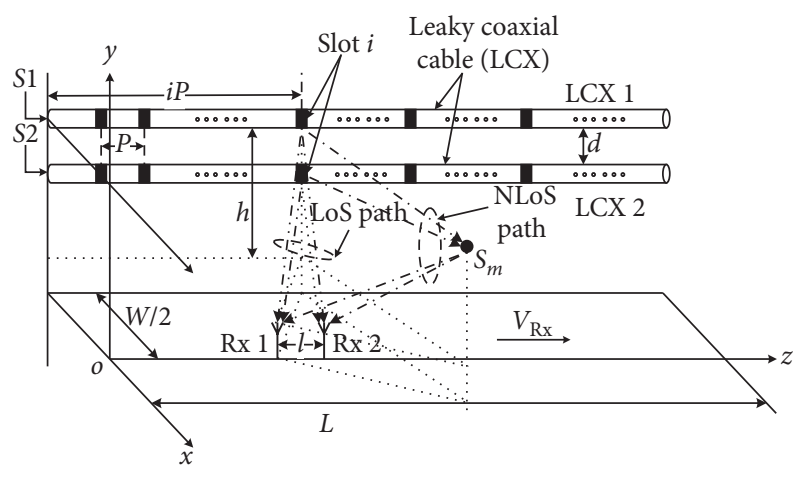

(c)

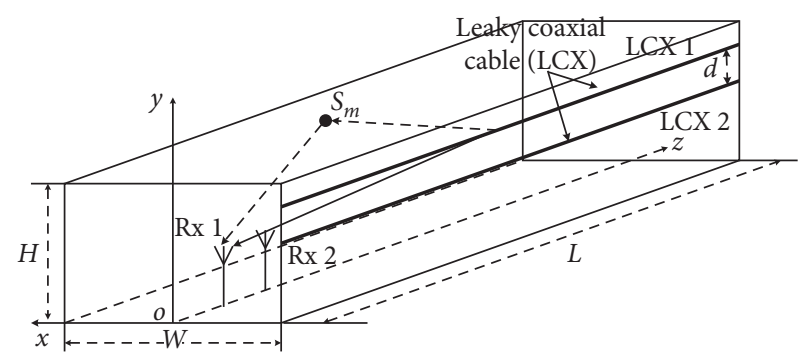

(b)

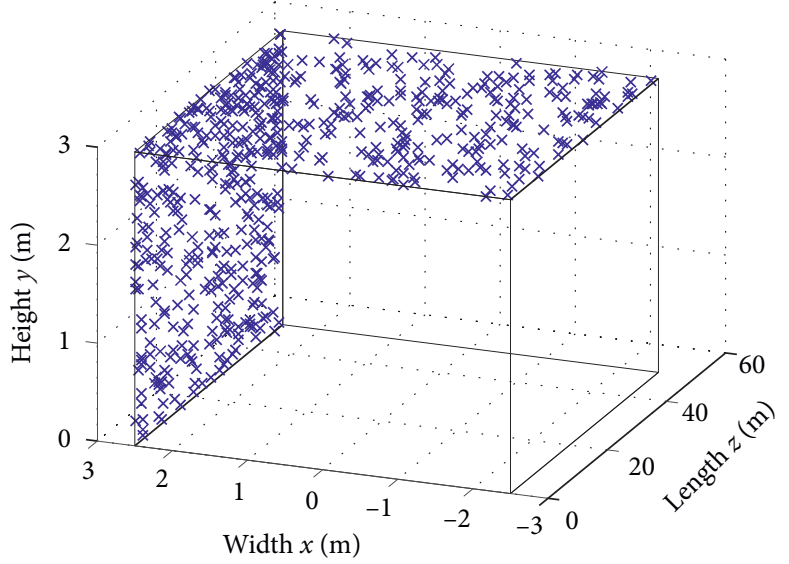

(d)

FIGURE 1: (a) Leaky coaxial cable (LCX) structure; (b) propagation scenario of MIMO using two LCXs in the tunnel; (c) nonstationary 3D

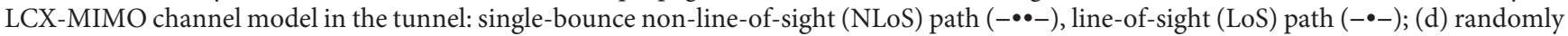
distributed scatters $(\times)$ on the wall and roof of a tunnel with length $L=50 \mathrm{~m}$, width $W=5 \mathrm{~m}$, and height $H=3 \mathrm{~m}$.

Zhongtian technology company (ZTT), and the longitudinal power attenuation constant of LCX is $\alpha=4.0 \mathrm{~dB} / 100 \mathrm{~m}$. It can be seen that $\alpha_{0}=10^{-\alpha P / 10 / 2 / 100} \approx 1$, so the influence of $\alpha_{i}$ on CIR is ignored in this paper. The phase variation of $i$-th slot of LCX and the propagation constant in LCX are $\beta_{i}$ and $k_{r}$, respectively, and it can be expressed as follows:

$$
\begin{aligned}
& \beta_{i}=\beta_{0} i, \\
& k_{r}=k_{0} \sqrt{\varepsilon_{r}},
\end{aligned}
$$

where $\beta_{0}=k_{r} P$ is the phase variation per unit slot in LCX; $\varepsilon_{r}$ is the dielectric constant in LCX; $k_{0}=2 \pi f / c$ is the propagation constant in free space; $f$ is the center frequency of input signal of LCX; $c$ is the velocity of light; and $\lambda$ is the wavelength.

For Figure 1, the definition of LCX-MIMO model parameters is shown in Table 1 . Because the surfaces of the sidewalls of the tunnel and the top of the tunnel are rough, we assume that there are a large number of scatterers at the sidewalls and the roof of the tunnel, and the number of effective and independent scatterers is $M$. So, there are both line-of-sight (LoS) and non-line-of-sight (NLoS) propagation paths in the propagation channel. The definition of LCX-MIMO model parameters is described in Table 1 . The propagation characteristics of the LCX-MIMO system can be described by $2 \times 2$ CIR matrix; that is, $\mathbf{H}(t)=\left[h_{q p}(t)\right]_{2 \times 2}$. Therefore, the CIR $h_{q p}(t)$ from the $p$-th LCX to the $q$-th receiving antenna is formed by vector superposition of components (LoS and NLoS), and it can be expressed as follows:

$$
\begin{aligned}
& h_{q p}^{\mathrm{LoS}}(t)= \sqrt{\frac{K_{q p} \Omega_{q p}}{K_{q p}+1}} \cdot \lim _{N \longrightarrow \infty} N^{-1 / 2} \sum_{i=1}^{N} e^{-j\left(2 \pi f d_{i, q p} / c+\beta_{i}\right)} \\
& \cdot e^{-j 2 \pi f_{D} t \cos \left(\theta_{R}^{i(q, p)}-\gamma_{R}\right) \cos \beta_{R}^{i(q, p)},} \\
& h_{q p}^{\mathrm{NLoS}}(t)=\sqrt{\frac{\Omega_{q p}}{K_{q p}+1}} \lim _{N \longrightarrow \infty}^{M \longrightarrow \infty}(N M)^{-1 / 2} \\
& \cdot \sum_{i=1}^{N} \sum_{m=1}^{M} g_{i m} e^{j\left[\varphi_{i m}-2 \pi f / c\left(d_{i m, p}+d_{i m, q}\right)-\beta_{i}\right]} \\
& \cdot e^{-j 2 \pi f_{D} t \cos \left(\theta_{R}^{i m(q, p)}-\gamma_{R}\right) \cos \beta_{R}^{i m(q, p)}} .
\end{aligned}
$$

In the expression of CIR, $\Omega_{q p}$ represents the transmission power from the $p$-th transmitting antenna to $q$-th receiving antenna, that is, $\Omega_{l p}=E\left[\left|h_{l p}(t)\right|^{2}\right] ; K_{q p}$ is Rice factor, that is, $K_{q p}=\left|h_{q p}^{\mathrm{LOS}}\right|^{2} / E\left[\left|h_{q p}^{\mathrm{NLOS}}\right|^{2}\right]$; and $g_{i m}$ is the amplitude of the wave scattered by $m$-th scatterer $S_{m}$ to receiving antenna so that $(N M)^{-1} \sum_{i=1}^{N} \sum_{m=1}^{M} E\left[g_{i m}^{2}\right]=1 \quad$ as $N \longrightarrow \infty$ and $M \longrightarrow \infty$. In this paper, it is assumed that $g_{i m}$ is a zero-mean Rayleigh random distribution variable. $\varphi_{i m}$ denotes the independent and identically distributed (i.i.d.) random phase shift introduced by $m$-th scatterer $S_{m}$, which is the uniform distribution over $[0,2 \pi)$. In order to guarantee that $\Omega_{q p}=E[\mid$ $\left.\left.h_{q p}(t)\right|^{2}\right]$, the CIR in the above formula needs to be satisfied $(N M)^{-1} \sum_{i=1}^{N} \sum_{m=1}^{M}\left[g_{i m}^{2}\right]=1$ as $N \longrightarrow \infty$ and $M \longrightarrow \infty$, 
TABLE 1: Definition of LCX-MIMO model parameters.

\begin{tabular}{|c|c|}
\hline Definition & Parameters \\
\hline Tunnel size $($ length $\times$ width $\times$ height $)$ & $L \times W \times H$ \\
\hline LCX spacing & $d$ \\
\hline Element spacing of $\mathrm{Rx}$ array & $l$ \\
\hline Coordinates of $i$-th slot of LCX 1 & $(-W / 2, h, i p)$ \\
\hline Coordinate of $\mathrm{Rx} 1$ & $\left(x_{0}, y_{0}, z_{0}\right)$ \\
\hline Coordinate of $m$-th $(m=1, \ldots, M)$ scatter $S_{m}$ & $\left(x_{m}, y_{m}, z_{m}\right)$ \\
\hline Length of LCX & $L$ \\
\hline Period of slot of LCX & $P$ \\
\hline Velocity of receiving antennas array & $v_{\mathrm{RX}}$ \\
\hline Maximum of Doppler frequency & $f_{D}$ \\
\hline Distance between $i$-th $(i=1, \ldots, N)$ slot of $p$-th $(p=1,2)$ LCX and $q$-th $(q=1,2)$ receiving antenna for LoS path & $d i, q p$ \\
\hline Distance between $i$-th slot of $p$-th LCX and $m$-th scatter in the tunnel & $\operatorname{dim}, p$ \\
\hline Distance between $m$-th scatter in tunnel and $q$-th receiving antenna & \\
\hline Azimuth angle of arrival (AAOA) of $\operatorname{LoS}$ path from $p$-th Tx antenna to $q$-th $\mathrm{Rx}$ antenna & $\theta_{R}^{i(q, p)}$ \\
\hline Elevation angle of arrival (EAOA) of LoS from $p$-th Tx antenna to $q$-th $\mathrm{Rx}$ antenna & $\beta_{R}^{i(q, p)}$ \\
\hline AAOA of NLoS from $p$-th Tx antenna to $q$-th Rx antenna & $\theta_{R}^{i m(q, p)}$ \\
\hline EAOA of NLoS from $p$-th Tx antenna to $q$-th Rx antenna & $\beta_{R}^{\text {im }(q, p)}$ \\
\hline
\end{tabular}

where $f_{D}$ denotes the maximum Doppler shift, that is, $f_{D}=v_{\mathrm{RX}} / \lambda$. The azimuth angle of velocity is $\gamma_{R}$. In this paper, we assumed that the receiving antenna moves forward along the $z$-axis. Therefore, according to Figure $1(\mathrm{c}), \gamma_{R}=0$.

\section{Channel T-CF and $K$-Factor}

In this paper, it is assumed that LoS and NLoS propagation paths are independent of each other, and the mean amplitude of CIR is zero. According to [13, 20-23], we consider the transmitter correlation and receiver correlation are independent of each other in this paper. Because the receiver correlation is influenced by the receive antennas spacing, we focus on the correlation between two LCXs in the Tx and the first receive antenna. In his paper, we set the independence between LoS path and NLoS path. Then, the channel T-CF between LCXs (i.e., T-CF between CIR $h_{11}(t)$ and CIR $h_{12}(t)$ ) can be expressed as follows:

$$
\begin{aligned}
\rho_{11,12}(t, \tau) & =\frac{E\left[h_{11}(t) h_{12}^{*}(t-\tau)\right]}{\sqrt{E\left[\left|h_{11}(t)\right|^{2}\right] E\left[\left|h_{12}(t)\right|^{2}\right]}} \\
& =\frac{E\left\{h_{11}^{\mathrm{LoS}}(t)\left[h_{12}^{\mathrm{LoS}}(t-\tau)\right]^{*}\right\}+E\left\{h_{11}^{\mathrm{NLoS}}(t)\left[h_{12}^{\mathrm{NLOS}}(t-\tau)\right]^{*}\right\}}{\sqrt{\Omega_{11} \Omega_{12}}} \\
& =\rho_{11,12}^{\mathrm{LoS}}(t, \tau)+\rho_{11,12}^{\mathrm{NLoS}}(t, \tau),
\end{aligned}
$$

where $(\bullet)^{*}$ is the complex conjugate, $\tau$ is time separation, $\rho_{11,12}^{\text {LoS }}(t, \tau)$ is the LoS component of T-CF between LCXs, and $\rho_{11,12}^{\mathrm{NLoS}}(t, \tau)$ is the NLoS component of T-CF between LCXs.
3.1. LoS Channel T-CF. According to formula (2a), the LoS component of T-CF between LCXs can be expressed as follows:

$$
\begin{aligned}
\rho_{11,12}^{\operatorname{LoS}}(t, \tau)= & \frac{E\left\{h_{11}^{\operatorname{LoS}}(t)\left[h_{12}^{\operatorname{LoS}}(t-\tau)\right]^{*}\right\}}{\sqrt{\Omega_{11} \Omega_{12}}} \\
= & \sqrt{\frac{K_{11} K_{12}}{\left(K_{11}+1\right)\left(K_{12}+1\right)}} \lim _{\longrightarrow \infty} N^{-1} \sum_{i=1}^{N} e^{-j 2 \pi f / c\left(d_{i, 11}-d_{i 12}\right)} \\
& \cdot e^{\left\{-j 2 \pi f_{D} t \cos \left(\theta_{R}^{i(1,1)}-\gamma_{R}\right)\left[\cos \beta_{R}^{i(1,1)}-\cos \beta_{R}^{i(1,2)}\right]-j 2 \pi f_{D} \tau \cos \left(\theta_{R}^{i(1,2)}-\gamma_{R}\right) \cos \beta_{R}^{i(1,2)}\right\}} .
\end{aligned}
$$


In order to simplify the T-CF of LoS, based on the geometric relationship described in Figure 1, it can be seen that $\cos \left(\theta_{R}^{i(1,2)}-\gamma_{R}\right) \cos \beta_{R}^{i(1,2)}=\left(z_{0}-i P\right) / d_{i, 12}$. In this paper, we consider $d_{i, 11}>d$ and $d_{i, 12}>>d$, so $\beta_{R}^{i(1,1)} \approx \beta_{R}^{i(1,2)}$. Then, the LoS T-CF can be expressed as follows:

$$
\begin{aligned}
\rho_{11,12}^{\operatorname{LoS}}(\tau)= & \sqrt{\frac{K_{11} K_{12}}{\left(K_{11}+1\right)\left(K_{12}+1\right)}} \lim _{N \longrightarrow \infty} N^{-1} \sum_{i=1}^{N} e^{-j(2 \pi f / c)\left(d_{i, 11}-d_{i, 12}\right)} \\
& \cdot e^{-j 2 \pi f_{D} \tau\left(z_{0}-i P\right) / d_{i, 12}} .
\end{aligned}
$$

where $d_{i, 11}$ is the distance between $i$-th slot of LCX1 and Rx1, $d_{i, 12}$ is the distance between $i$-th slot of LCX 2 and Rx 1 , and they are given as

$$
\begin{aligned}
& d_{i, 11}=\sqrt{\left(x_{0}+\frac{W}{2}\right)^{2}+\left(y_{0}-h\right)^{2}+\left(z_{0}-i P\right)^{2}}, \\
& d_{i, 12}=\sqrt{\left(x_{0}+\frac{W}{2}\right)^{2}+\left(y_{0}-h+d\right)^{2}+\left(z_{0}-i P\right)^{2}} .
\end{aligned}
$$

3.2. NLoS Channel T-CF. According to (2b), the NLoS component of T-CF between LCXs can be expressed as follows:

$$
\begin{aligned}
\rho_{11,12}^{\mathrm{NLoS}}(t, \tau)= & \frac{E\left\{h_{11}^{\mathrm{NLoS}}(t)\left[h_{12}^{\mathrm{NLoS}}(t-\tau)\right]^{*}\right\}}{\sqrt{\Omega_{11} \Omega_{12}}}, \\
= & \frac{1}{\sqrt{\left(K_{11}+1\right)\left(K_{12}+1\right)}} \lim _{M \longrightarrow \infty}^{N \longrightarrow \infty}(N M)^{-1} \sum_{i=1}^{N} \sum_{m=1}^{M} E\left[g_{i m}^{2}\right] \\
& \cdot e^{-j 2 \pi f / c\left(d_{i m, \mathrm{Tx} 1}-d_{i m, \mathrm{~T} 2}\right)} \cdot e^{-j 2 \pi f_{D} \tau \cos \left(\theta_{R}^{i m(1,2)}-\gamma_{R}\right) \cos \beta_{R}^{i m(1,2)}}
\end{aligned}
$$

where $d_{i m, \mathrm{Tx} 1}$ is the distance between i-th slot of LCX1 (i.e., Tx1) and $m$-th scatterer $S_{m}, d_{i m, \mathrm{Tx} 2}$ is the distance between i-th slot of LCX2 (i.e., Tx2) and $m$-th scatterer $S_{m}, d_{i m, \mathrm{Rx} 1}$ is the distance between $m$-th scatterer $S_{m}$ and Rx1, and they are given as

$$
\begin{aligned}
& d_{i m, \mathrm{Tx} 1}=\sqrt{\left(x_{m}+\frac{W}{2}\right)^{2}+\left(y_{m}-h\right)^{2}+\left(z_{m}-i P\right)^{2}}, \\
& d_{i m, \mathrm{Tx} 2}=\sqrt{\left(x_{m}+\frac{W}{2}\right)^{2}+\left(y_{m}-h+d\right)^{2}+\left(z_{m}-i P\right)^{2}}, \\
& d_{i m, \mathrm{Rx} 1}=\sqrt{\left(x_{0}-x_{m}\right)^{2}+\left(y_{0}-y_{m}\right)^{2}+\left(z_{0}-z_{m}\right)^{2}} .
\end{aligned}
$$

In order to simplify the T-CF of LoS, based on the geometric relationship described in Figure 1, it can be seen that $\cos \left(\theta_{R}^{i m(1,2)}-\gamma_{R}\right) \cos \beta_{R}^{i m(1,2)}=\left(z_{0}-z_{m}\right) / d_{i m, R \times 1}$. Then, the NLoS T-CF can be expressed as follows: 


$$
\begin{aligned}
\rho_{11,12}^{\mathrm{NLoS}}(\tau)= & \frac{1}{\sqrt{\left(K_{11}+1\right)\left(K_{12}+1\right)}} \lim _{\substack{M \longrightarrow \infty \\
\longrightarrow}}(N M)^{-1} \sum_{i=1}^{N} \sum_{m=1}^{M} E\left[g_{i m}^{2}\right] \\
& \cdot e^{-j 2 \pi f / c\left(d_{i m, \mathrm{Tx} 1}-d_{i m, \mathrm{Tx} 2}\right)} \cdot e^{-j 2 \pi f_{D} \tau\left(z_{0}-z_{m}\right) / d_{i m, \mathrm{Rx} 1}} .
\end{aligned}
$$

3.3. K-Factor. The Rice $K$-factor is usually used in evaluating the communication link quality, such as capacity and performance of the MIMO system. Therefore, the estimation of the $K$-factor is very important in different wireless scenarios. With fixed $S N R$ level, the higher $K$-factor means larger spatial correlation and smaller channel capacity. Usually, we can estimate $K$-factor based on the measurement data for the MIMO channel. For a complex channel gain matrix $\mathbf{H}(t)$, the $K$-factor can be estimated as [24]

$$
K_{[d B]}=\frac{\varepsilon[|\mathbf{H}(t)|]^{2}}{2 \operatorname{var}(|\mathbf{H}(t)|)}
$$

where $|\bullet|$ denotes the operation of matrix determinant, $\varepsilon$ denotes the mean value of $|\mathbf{H}(t)|$, var denotes the variance of $|\mathbf{H}(t)|$.

\section{Experiment Results and Analysis}

4.1. Parameter Setup. In this paper, an experiment was carried out in a tunnel-like scenario at Zhongtian Technology Company (ZTT), Nantong, China. In Figure 2, the overall length of the tunnel is $100 \mathrm{~m}$, and the rectangular part and arched part of the tunnel are $50 \mathrm{~m}$, respectively. In this paper, the rectangular part has been considered in the experiment. As shown in Figure 2, it is the experiment scenario description for Nantong tunnel; Figure 2(a) is a photograph of the tunnel; Figure 2(b) is the front view of the tunnel; Figure 2(c) is experiment configuration of LCX-MIMO system; and Figure 2(d) is the top view of the tunnel. And the description of other parameters in the experiment is shown in Table 2.

4.2. Results and Analysis. In this section, we estimated the $K$-factor using formula (10) to guarantee that the $K$-factor of the theory is consistent with the experiment. Figure 3 describes the cumulative distribution function (CDF) of the $\mathrm{K}$-factor in the experiment, and we can see that the average value of the $K$-factor is $4.032 \mathrm{~dB}$. Therefore, we set the theoretical value of the $K$-factor as $4 \mathrm{~dB}$. The statistics of the $K$-factor in measurement is shown in Table 3.

\section{Simulation Results and Validation}

In this section, firstly, we compare the theoretical and simulation results of T-CF to further verify the reasonability of the channel model proposed in this paper. Secondly, we will show that the performance of the LCX-MIMO system is better by comparing the T-CF of the dipole MIMO system with the T-CF of the LCX-MIMO system. According to (3)-(9), the Monte-Carlo method is used in simulating the T-CF $(\mathrm{LoS}+\mathrm{NLoS})$ of different time separations $\tau$, different $\mathrm{K}$-factors, and different maximum Doppler shifts $f_{\mathrm{D}}$ (i.e., $\left.f_{D}=v_{\mathrm{RX}} / \lambda\right)$. Because the LoS component of CIR is deterministic, and NLoS component of CIR is a random process, we can multiply the iteration NLoS component of CIR by the Monte-Carlo method. In this paper, we set the number of iteration as $2 \times 10^{4}$.

5.1. Parameter Setup. Here, we assume that the scatterers are randomly distributed on the sidewall of the tunnel and the roof of the tunnel as shown in Figure 1(d). The simulation scenario of LCX-MIMO is the same as Figure 1. The simulation propagation scheme of the MIMO system is shown in Figure 4. For the LCX-MIMO system, Tx is two LCXs and $\mathrm{Rx}$ is two dipole antennas. For the dipole MIMO system, the Tx and Rx are both two dipole antennas. These two systems have the same parameters configuration at the same scenario. For the LCX-MIMO system and dipole MIMO system, the Tx antennas have the same $x$ coordinate and $y$ coordinate, but the $z$ coordinate of Tx antennas for dipole MIMO is $0 \mathrm{~m}$. The $\mathrm{Rx}$ array moves along the $z$-axis. The number of tunnel wall scatters and tunnel roof scatters is assumed $M=500$. The other parameters are the same as Section 4 .

5.2. Results and Analysis. Here, based on (3), (5), and (7), the numerical results are obtained by simulation for the T-CF of LCX-MIMO channel model in rectangular tunnel scenario.

Figure 5 presents the T-CF for various LCX spacing $d$ with different $K$-factor (here, we assume $K_{11}=K_{12}$ ) when time separation $\tau=1 \mathrm{~ns}, f_{D}=600 \mathrm{~Hz}$ (i.e., $v_{\mathrm{RX}}=360 \mathrm{~km} / \mathrm{h}$ ), $l=0.5 \mathrm{~m}$. As we can see in Figure 5, the T-CF decreases with the LCX spacing increasing, and the T-CF is higher when $K$-factor is larger. Meanwhile, the theoretical value of T-CF matches very well with the simulation value of T-CF. It shows that the model proposed in this paper is reasonable. In the MIMO system, the LoS path has a negative effect on the channel capacity. The $K$-factor is the ratio between the power of LoS path and the power of NLoS path. Therefore, the $K$-factor is larger when the power of LoS path is higher. Finally, the T-CF is higher when $K$-factor is larger.

Figure 6 shows the T-CF for different time separation $\tau$ with various Doppler shifts $f_{D}[150 \mathrm{~Hz}(90 \mathrm{~km} / \mathrm{h}), 300 \mathrm{~Hz}$ $(180 \mathrm{~km} / \mathrm{h}), 600 \mathrm{~Hz}(360 \mathrm{~km} / \mathrm{h})]$ of $\mathrm{Rx}$ when the LCX spacing $d=4.8 \lambda$, Rice factor $K_{11}=K_{12}=4 \mathrm{~dB}$, and element spacing of $\mathrm{Rx}$ array $l=0.5 \mathrm{~m}$. As shown in Figure 6, the T-CF of theory 


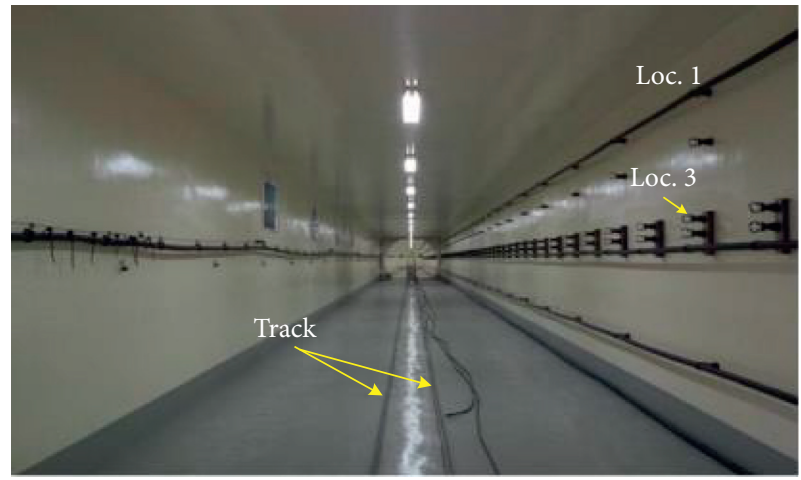

(a)

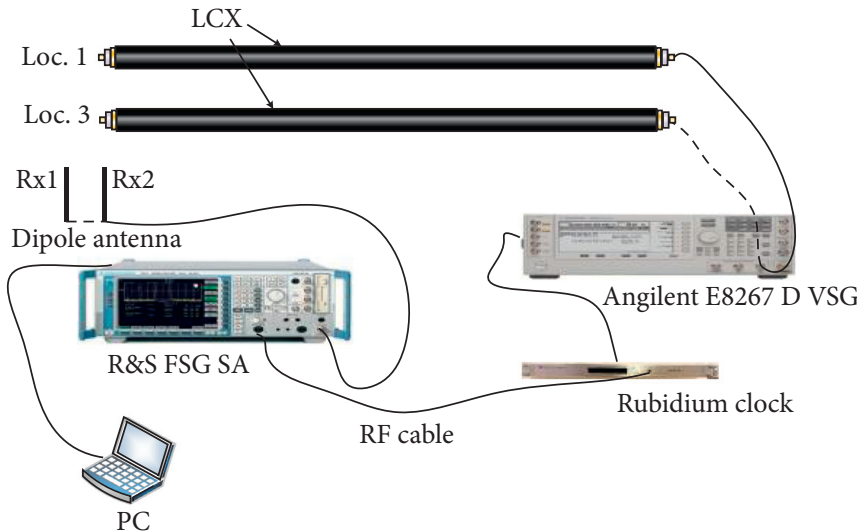

(c)

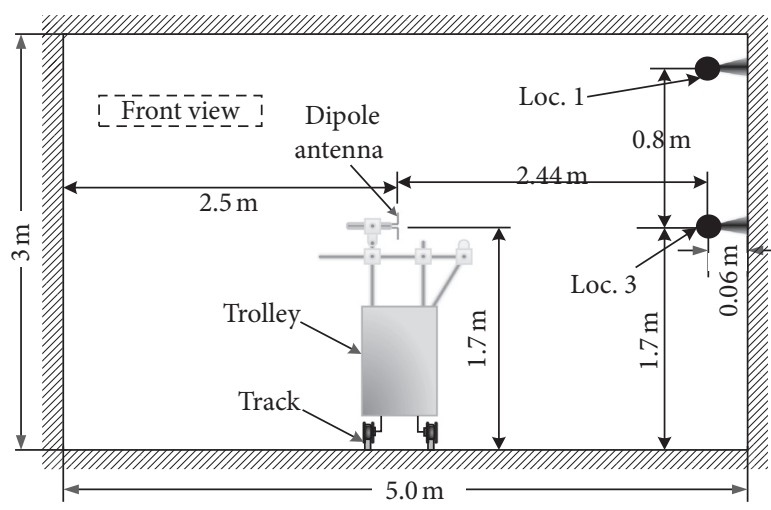

(b)

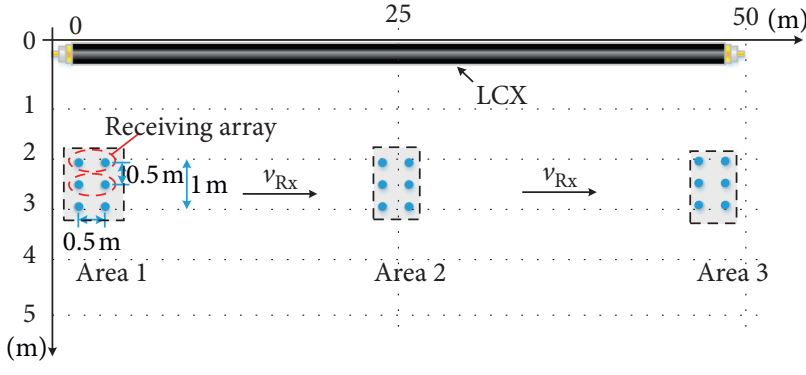

- Dipole antenna

Figure 2: Experiment scenario description of Nantong tunnel: (a) photograph of the tunnel; (b) the front view of the tunnel; (c) experiment configuration of LCX-MIMO system; (d) top view of the tunnel.

TABLE 2: Experiment parameters.

\begin{tabular}{lc}
\hline Parameter & Value \\
\hline Carrier frequency $\left(f_{\mathrm{c}}\right)$ & $1.8 \mathrm{GHz}$ \\
Velocity of light & $3 \times 10^{8} \mathrm{~m} / \mathrm{s}$ \\
Wavelength of carrier frequency $(\lambda)$ & $0.167 \mathrm{~m}$ \\
Bandwidth & $40.8 \mathrm{MHz}$ \\
Sampling rate & $81.6 \mathrm{MHz}$ \\
Tx antennas array & $50 \mathrm{~m}$ ZTT LCX-horizontal polarization \\
Rx antennas array & UHA9125D dipole antenna \\
LCX spacing $(d)$ & $0.8 \mathrm{~m}(4.8 \lambda)$ \\
Velocity of $R x$ & $360 \mathrm{~km} / \mathrm{h}$ \\
Size of tunnel $(L \times W \times H)$ & $50 \times 5 \times 3\left(\mathrm{~m}^{3}\right)$ \\
Period of LCX slots $(P)$ & $0.25 \mathrm{~m}$ \\
Element spacing of Rx array $(l)$ & $0.5 \mathrm{~m}$ \\
\hline
\end{tabular}

TABle 3: Statistics of $K$-factor.

\begin{tabular}{lccc}
\hline Meas. scenario & Min $(\mathrm{dB})$ & Max $(\mathrm{dB})$ & Mean $(\mathrm{dB})$ \\
\hline Rectangular tunnel & 2.4571 & 7.7483 & 4.032 \\
\hline
\end{tabular}




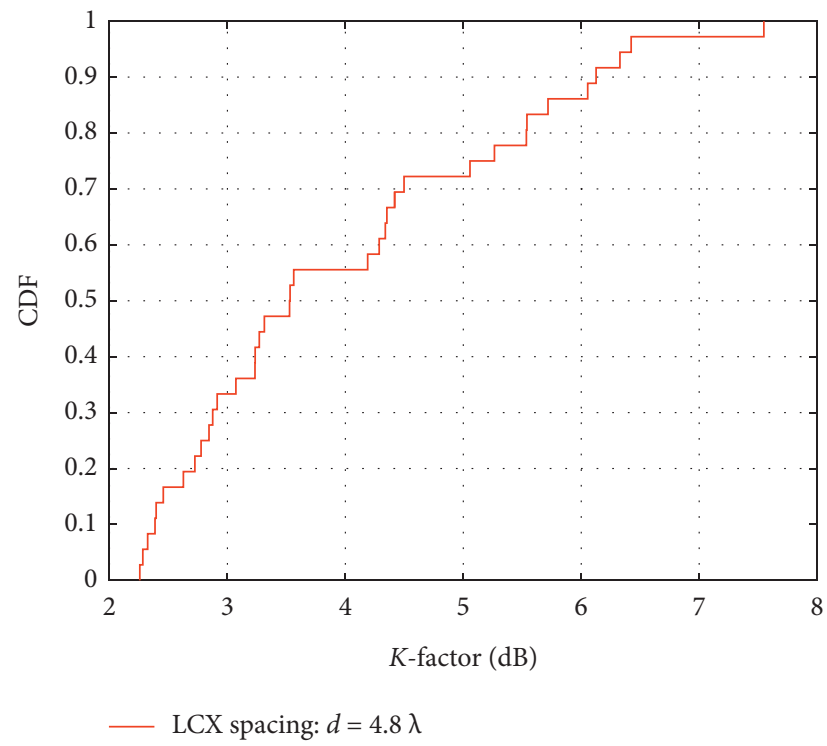

Figure 3: Cumulative distribution function (CDF) of $K$-factor in the experiment.

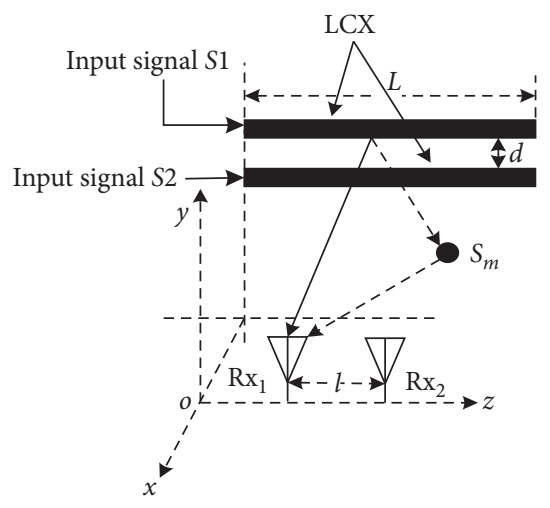

(a)

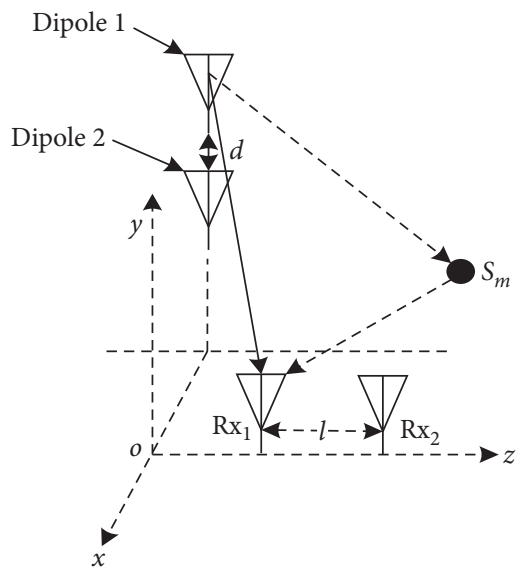

(b)

FIGURE 4: Simulation propagation scheme of MIMO system: (a) LCX-MIMO and (b) dipole MIMO.

matches with the T-CF of simulation very well. It is obvious that the T-CF decreases with increasing time separation $\tau$. The T-CF decreases more rapidly when Doppler shift $f_{\mathrm{D}}$ increases. As shown in $[16,18]$, their results show that the movement velocity of the moving vehicles will affect the $\mathrm{V} 2 \mathrm{~V}$ channel characteristics of the MIMO system.

In Figure 7, it shows the T-CF of dipole MIMO system and the T-CF of LCX-MIMO system for different Doppler shifts $f_{D}[150 \mathrm{~Hz}(90 \mathrm{~km} / \mathrm{h}), 300 \mathrm{~Hz}(180 \mathrm{~km} / \mathrm{h}), 600 \mathrm{~Hz}$
$(360 \mathrm{~km} / \mathrm{h})]$ of $\mathrm{Rx}$ when the LCX spacing $d=4.8 \lambda$, Rice factor $K_{11}=K_{12}=4 \mathrm{~dB}$, and element spacing of Rx array $l=0.5 \mathrm{~m}$. By comparing the T-CF between dipole MIMO system and LCX-MIMO system, we can see that the T-CF of LCX-MIMO decreases more rapidly than T-CF of dipole MIMO with different time separation $\tau$ when Doppler shift $f_{D}$ increases. It means that the performance of LCXMIMO is better than the performance of dipole MIMO in the tunnel. 


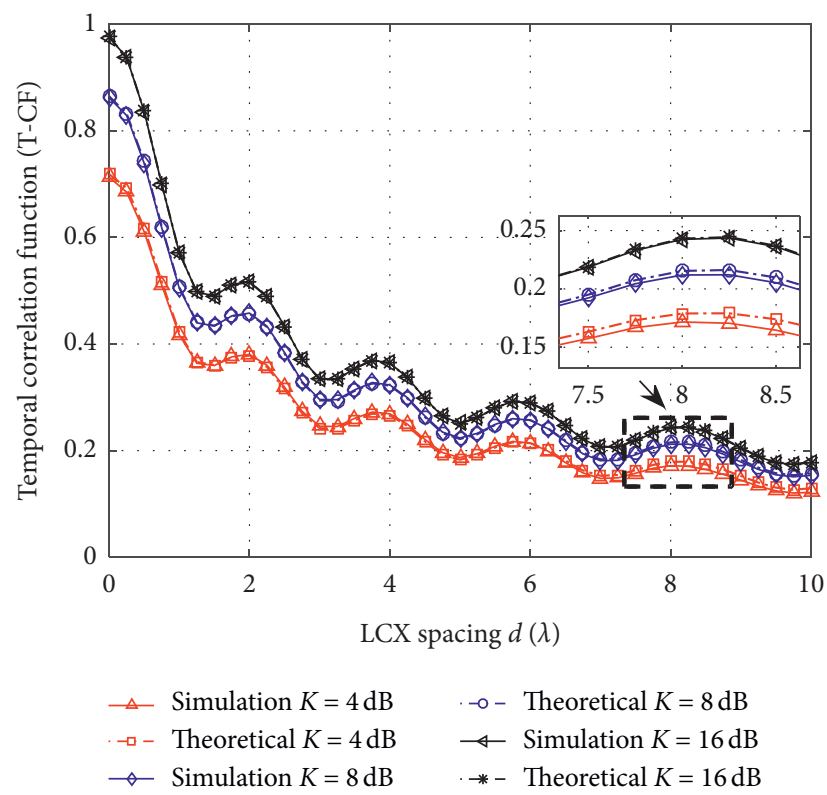

FIgURE 5: T-CF of LCX-MIMO for various LCX spacing with different $K$-factors $\left(K_{11}=K_{12}\right)$, time separation $\tau=1 \mathrm{~ns}, f_{D}=600 \mathrm{~Hz}$ (i.e., $v_{\mathrm{RX}}=360 \mathrm{~km} / \mathrm{h}$ ), and $l=0.5 \mathrm{~m}$.

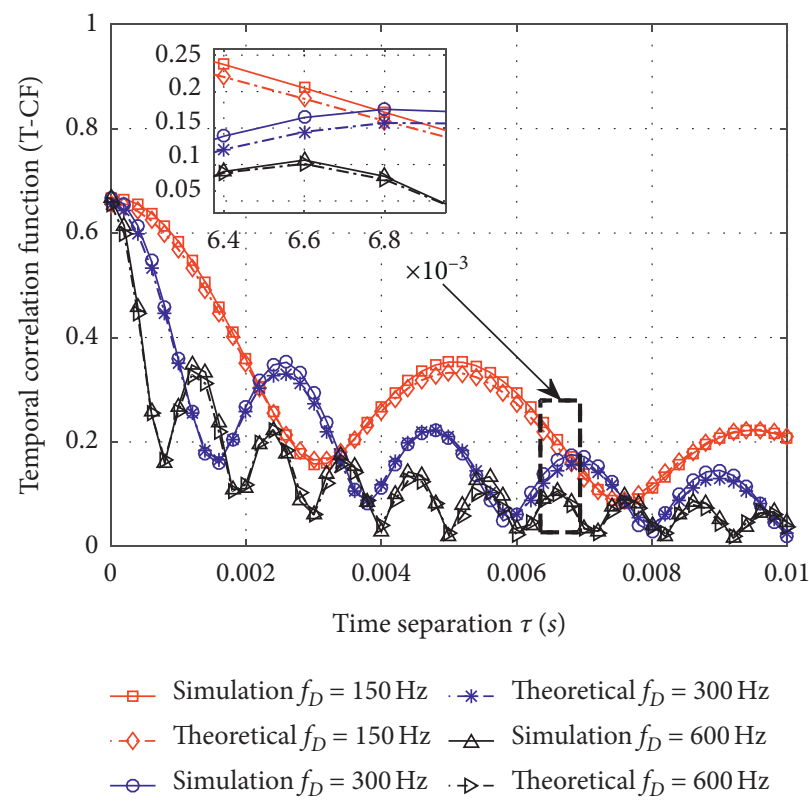

FIGURE 6: T-CF of LCX-MIMO for different time separation $\tau$ with different Doppler shifts $f_{D}[150 \mathrm{~Hz}(90 \mathrm{~km} / \mathrm{h}), 300 \mathrm{~Hz}(180 \mathrm{~km} / \mathrm{h}), 600 \mathrm{~Hz}$ $(360 \mathrm{~km} / \mathrm{h})]$ of the receiver and $d=4.8 \lambda, K_{11}=K_{12}=4 \mathrm{~dB}, l=0.5 \mathrm{~m}$. 


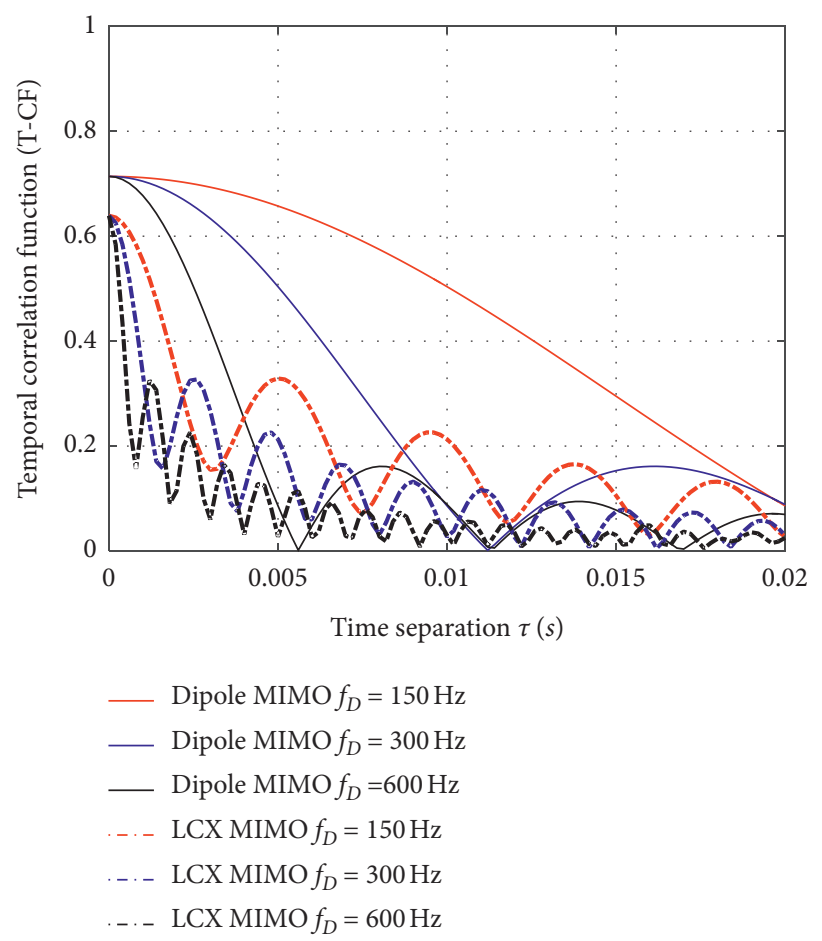

Figure 7: T-CF of dipole MIMO and T-CF of LCX-MIMO with different Doppler shifts $f_{D}[150 \mathrm{~Hz}(90 \mathrm{~km} / \mathrm{h}), 300 \mathrm{~Hz}(180 \mathrm{~km} / \mathrm{h}), 300 \mathrm{~Hz}$ $(360 \mathrm{~km} / \mathrm{h})]$ of the receiver and $d=4.8 \lambda, K_{11}=K_{12}=4 \mathrm{~dB}, l=0.5 \mathrm{~m}$.

\section{Conclusion}

In this paper, we have proposed a 3D nonstationary LCXMIMO channel model with a high-mobility wireless communication system using LCX in a rectangular tunnel environment. The effective scatterers of the tunnel are considered to be randomly distributed on the roof and the sidewalls of the tunnel in this model. Taking into account single-bounce scattering under LoS and NLoS propagation condition, we derived the analytical expressions of CIR and T-CF. In order to verify the channel model proposed in this paper, the simulation is carried out in a tunnel-like scenario.

From the numerical results, we conclude that the T-CFs is significantly affected by time separation, K-factor, movement velocity of $\mathrm{Rx}$, and LCX spacing. Finally, by comparing the T-CF between dipole MIMO system and LCX-MIMO system, we can see that the T-CF of LCXMIMO decreases more rapidly than that of dipole MIMO with different time separation $\tau$ when Doppler shift $f_{D}$ increases, which proves that the performance of LCX-MIMO system is better than that of the dipole MIMO system.

\section{Data Availability}

The measured data used to support the findings of this study have not been made available because the research findings will be commercialized. The simulation data is obtained by the special software purchased by our laboratory. This paper uses MATLAB software to process the data results.

\section{Conflicts of Interest}

The authors declare that they have no conflicts of interest.

\section{Acknowledgments}

This work was supported by the National Natural Foundation of China under Grant 61871261 and Project of Shanghai Science and Technology Commission 18511105704. The author particularly appreciates Mr. Asad Saleem for his help during checks and modifications of this paper.

\section{References}

[1] Y. Wu, G. Zheng, and T. Wang, "Performance analysis of MIMO transmission scheme using single leaky coaxial cable," IEEE Antennas and Wireless Propagation Letters, vol. 16, pp. 298-301, 2017.

[2] Y. Wu, G. Zheng, A. Saleem, and Y. P. Zhang, "An experimental study of MIMO performance using leaky coaxial cables in a tunnel," IEEE Antennas and Wireless Propagation Letters, vol. 16, pp. 1663-1666, 2017.

[3] J. H. Wang and K. K. Mei, "Theory and analysis of leaky coaxial cables with periodic slots," IEEE Trans. Antennas and Propag.vol. 49, no. 12, pp. 1723-1732, 2001.

[4] J. Medbo and A. Nilsson, "Leaky coaxial cable MIMO performance in an indoor office environment," in Proceedings of the IEEE International Symposium on Personal, indoor and Mobile Radio Communications-(PIMRC), pp. 2061-2066, Sydney, Australia, September 2012. 
[5] Y. Hou, S. Tsukamoto, S. Li, T. Higashino, K. Kobayashi, and M. Okada, "Capacity evaluation of MIMO channel with one leaky coaxial cable used as two antennas over linear-cell environments," IEEE Transactions on Vehicular Technology, vol. 66, no. 6, pp. 4636-4646, June 2017.

[6] Y. Hou and S. Tsukamoto, "2 by 2 MIMO system using single leaky coaxial cable for linear-cells," in Proceedings of the IEEE 25th Annual International Symposium on Personal, indoor and Mobile Radio Communications-(PIMRC), pp. 327-331, Washington, DC, USA, September 2014.

[7] Y. Hou and S. Tsukamoto, "Performance comparison for 2 by 2 MIMO system using single leaky coaxial cable over WLAN frequency band," in Proceedings of the Signal and Information Processing Association Annual Summit and Conference (APSIPA), pp. 1-5, Chiang Mai, Thailand, December 2014.

[8] S. Li and Y. Hou, "A study of capacity of 4-by-4 MIMO channel using leaky coaxial cables for linear-cells," in Proceedings of the IEEE 15th International Symposium on Communications and Information Technologies (ISCIT), pp. 7-9, Nara, Japan, October 2015.

[9] Y. Hou, S. Tsukatomo, T. Meada et al., "Realization of 4-by-4 MIMO channel using onecomposite leaky coaxial cable," in Proceedings of the IEEE 12th Annual IEEE Consumer Communications and Networking Conference (CCNC), pp. 9-12, Las Vegas, ND, USA, September 2015.

[10] Y. Hou, S. Tsukatomo, M. Ariyoshi et al., "4-by-4 MIMO channel using two leaky coaxial cables (LCXs) for wireless applications over linear-cell," in Proceedings of the IEEE 3rd Global Conference on Consumer Electronics (GCCE), pp. 125-126, Tokyo, Japan, July 2014.

[11] K. Zhang, F. Zhang, G. Zheng, and A. Saleem, "GBSB model for MIMO channel using leaky coaxial cables in tunnel," IEEE Access, vol. 7, pp. 67646-67655, 2019.

[12] K. Zhang, F. Zhang, and G. Zheng, "Space-time correlation for three-dimensional MIMO channel model using leaky coaxial cable in rectangular tunnel," International Journal of Antennas and Propagation, vol. 2020, Article ID 3984148, 9 pages, 2020.

[13] A. Abdi and M. Kaveh, "A space-time correlation model for multielement antenna systems in mobile fading channels," IEEE Journal on Selected Areas in Communications, vol. 20, no. 3, pp. 550-560, 2002.

[14] A. Abdi and M. Kaveh, "Space- time correlation modeling of multielement antenna system in mobile fading channels," in Proceedings of the IEEE International Conference on Acoustics, Speech, and Signal Processing, pp. 2505-2508, Salt Lake City, UT, USA, May 2001.

[15] G. J. Byers and F. Takawira, "Spatially and temporally correlated MIMO channels: modeling and capacity analysis," IEEE Transactions on Vehicular Technology, vol. 53, no. 3, pp. 634-643, 2004.

[16] M. Patzold, B. Hogstad, and N. Youssef, "Modeling, analysis, and simulation of MIMO mobile-to-mobile fading channels," IEEE Transactions on Wireless Communications, vol. 7, no. 2, pp. 510-520, 2008.

[17] Q. Zhu, Y. Yang, C.-X. Wang et al., "Spatial correlations of a 3-D non-stationary MIMO channel model with 3-D antenna arrays and 3-D arbitrary trajectories," IEEE Wireless Communications Letters, vol. 8, no. 2, pp. 512-515, 2019.

[18] H. Jiang, Z. Zhang, L. Wu, and J. Dang, "Three-dimensional geometry-based UAV-MIMO channel modeling for A2G communication environments," IEEE Communications Letters, vol. 22, no. 7, pp. 1438-1441, 2018.
[19] X. Cheng and Y. Li, "A 3-D geometry-based stochastic model for UAV-MIMO wideband nonstationary channels," IEEE Internet of Things Journal, vol. 6, no. 2, pp. 1654-1662, 2019.

[20] H. Jiang, Z. Zhang, L. Wu, J. Dang, and G. Gui, "A 3-D nonstationary wideband geometry-based channel model for MIMO vehicle-to-vehicle communications in tunnel environments," IEEE Transactions on Vehicular Technology, vol. 68, no. 7, pp. 6257-6271, 2019.

[21] N. Avazov, S. M. R. Islam, D. Park, and K. S. Kwak, "Statistical characterization of a 3-D propagation model for $\mathrm{V} 2 \mathrm{~V}$ channels in rectangular tunnels," IEEE Antennas and Wireless Propagation Letters, vol. 16, pp. 2392-2395, 2017.

[22] N. Avazov and M. Patzold, "A novel wideband MIMO car-tocar channel model based on a geometrical semi-circular tunnel scattering model," IEEE Transactions on Vehicular Technology, vol. 65, no. 3, pp. 1070-1082, 2016.

[23] H. Zheng and X. Nie, "GBSB model for MIMO channel and its space-time correlation analysis in tunnel," in Proceedings of the IEEE International Symposium on Knowledge Acquisition and Modeling Workshop, pp. 221-224, Wuhan, China, December 2008.

[24] I. B. Mabrouk, J. Hautcoeur, L. Talbi et al., "Feasibility of a millimeter-wave MIMO system for short-range wireless communications in an underground gold mine," IEEE Transactions on Antennas and Propagation, vol. 61, no. 8, pp. 4296-4304, 2013. 